\title{
INVARIANT MEASURES ON LOCALLY COMPACT SEMIGROUPS
}

\begin{abstract}
ROGER RIGELHOF
Abstract. The main result of this paper shows that a locally compact abelian semigroup is embeddable as an open subsemigroup of a locally compact abelian group $G$ if and only if the translations $x \mapsto x+y$ are open maps and there exists a nonnegative regular measure $\mu$ on $S$ such that $\mu(U)=\mu(x+U)>0$ for every open set $U$ and $x$ in $S$.
\end{abstract}

Our main result is a somewhat stronger statement than the above in that we show that whenever such a measure exists it is the restriction to $S$ of the Haar measure on $G$. This provides a partial answer to a question raised by $\mathrm{J}$. $\mathrm{H}$. Williamson in $[5, \S 5]$. We follow the terminolozy of [5] as regards semigroups and the measure theoretic terminology of [3]. In particular:

A locally compact abelian semigroup $S$ is an abelian semigroup (not necessarily having a unit) which is a locally compact Hausdorff space such that for each $y$ in $S$ the map $x \mapsto x+y$ is continuous. We say that a locally compact abelian semigroup $S$ is embeddable in a locally compact group $G$ if there exists a bicontinuous semigroup monomorphism $\phi$ mapping $S$ into $G$. The following proposition is of independent interest (see [4, Theorem 2.1 and Lemma 1.3]).

Proposition. Let $S$ be a locally compact abelian semigroup. The following conditions on $S$ are equivalent.

(1) $S$ is a cancellation semigroup and for each open subset $U$ of $S$, $x+U$ is open for each $x$ in $S$.

(2) $S$ is embeddable as an open subsemigroup of a locally compact group $G$.

Proof. It is clear that (2) implies (1). To show that (1) implies (2) let $R$ be the equivalence relation on $S \times S$ defined by $(x, y) R\left(x_{0}, y_{0}\right)$ if and only if $x+y_{0}=y+x_{0}$. It is well known and easy to show that $G=S \times S / R$ is an abelian group. For $x$ in $S$ let $\phi(x)$ be the equivalence class $\{(x+y, y): y \in S\}$. The map $\phi: x \mapsto \phi(x)$ is one-one and satisfies $\phi(x+y)=\phi(x)+\phi(y)$. We now define a topology on $G$. For $x$ in $S$ let $\mathbb{B}(x)$ be the neighbourhood filter of $x$. Choose some $x_{0}$ in $S$ and for

Received by the editors March 11, 1970.

AMS 1969 subject classifications. Primary 2875, 4250; Secondary 2205.

Key words and phrases. Invariant measures, Haar measures, locally compact abelian semigroups, topological semigroups.

Copyright (c) 1971, American Mathematical Society 
each $x$ in $G$ let $x \cdot B$ be the filter on $G$ generated by the filter base $\left\{\phi(U)-\phi\left(x_{0}\right)+x: U \in \beta\left(x_{0}\right)\right\}$. We first show that if $x \in S$, then $\phi(x) \cdot B$ is generated by the filter base $\{\phi(U): U \in \mathbb{B}(x)\}$. Given $U \in B\left(x_{0}\right), U+x \in B\left(x_{0}+x\right)$ and the continuity of $z \mapsto z+x_{0}$ at $x$ means there is a $V \in B(x)$ with $V+x_{0} \subset U+x$ and so $\phi(V) \subset \phi(U)$

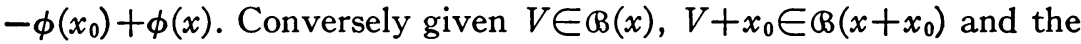
continuity of $z \mapsto z+x$ at $x_{0}$ means there is a $U \in B\left(x_{0}\right)$ such that $U+x \subset V+x_{0}$ and so $\phi(U)-\phi\left(x_{0}\right)+\phi(x) \subset \phi(V)$. Therefore $\phi(x) \cdot \beta$ is generated by $\{\phi(U): U \in B(x)\}$. We now show that there is a unique topology on $G$ such that for each $x$ in $G, x \cdot B$ is the neighbourhood filter of $x$. For this it is sufficient by [1, Chapitre $1, \S 1$, No. 1] to show for each $x$ in $G$ :

(i) if $V \in x \cdot B$ then $x \in V$;

(ii) if $V \in x \cdot B$ then there is a $W \in x \cdot B$ such that $y \in W$ implies $V \in y \cdot B$.

Clearly (i) is satisfied, so we show (ii). Let $V \in x \cdot B$. Then there is an open neighbourhood $U$ of $x_{0}$ such that $W=\phi(U)-\phi\left(x_{0}\right)+x \subset V$. If $y \in W$, there is a $u \in U$ with $y=\phi(u)-\phi\left(x_{0}\right)+x$ and there is a $V^{\prime} \in B\left(x_{0}\right)$ such that $V^{\prime}+u \subset U+x_{0}$. Thus

$$
\begin{gathered}
\phi\left(V^{\prime}\right)-\phi\left(x_{0}\right)+y=\phi\left(V^{\prime}\right)-\phi\left(x_{0}\right)+\phi(u)-\phi\left(x_{0}\right)+x \\
\subset \phi(U)-\phi\left(x_{0}\right)+x=W
\end{gathered}
$$

and therefore $W \in y \cdot ß$ so that (ii) is satisfied.

It is clear that $G$ with this topology is a Hausdorff space and that the maps $x \mapsto x+y$ are continuous. Moreover for each $x$ in $S, \phi(x) \cdot B$ is generated by $\{\phi(U): U \in \mathbb{B}(x)\}$ so that $\phi$ is a topological embedding and $\phi(S)$ is an open subset of $G$. The continuity and openness of the map $x \mapsto x+y$ for each $y$ in $G$ together with the local compactness of $S$ imply that $G$ is locally compact. Thus $G$ is a locally compact semigroup which is a group. A theorem of R. Ellis [2, Theorem 2] shows that $G$ is a locally compact group. This completes the proof.

THEOREM. Let $S$ be a locally compact abelian semigroup and $\mu a$ nonnegative regular measure on $S$. Suppose that $S$ and $\mu$ satisfy the following condition.

$\left({ }^{*}\right)$ For each open set $U, x+U$ is open for each $x$ in $S$ and $\mu(x+U)$ $=\mu(U)>0$.

Then $S$ is embeddable as an open subsemigroup in a locally compact abelian group $G$ and $\mu$ is the restriction of the Haar measure of $G$ to $S$. Conversely if $S$ is an open subsemigroup of a locally compact abelian group $G$, and if $\mu$ is the restriction to $S$ of the Haar measure of $G$, then $S$ is a locally compact abelian semigroup and $S$ and $\mu$ satisfy condition $\left({ }^{*}\right)$. 
Proof. First suppose $S$ and $\mu$ are given and satisfy $\left(^{*}\right)$. We begin by showing that $S$ is a cancellation semigroup. If not there are $x, y z$ in $S$ such that $y+x=y+z$ and $x \neq z$. There are open relatively compact neighbourhoods $U$ of $x$ and $V$ of $z$ such that $U \cap V$ is empty. Now

$$
\begin{aligned}
\mu((y+U) \cup(y+V)) & =\mu(y+(U \cup V))=\mu(U \cup V) \\
& =\mu(U)+\mu(V)=\mu(y+U)+\mu(y+V) .
\end{aligned}
$$

Since regular measures are by definition finite on compacta it follows that $\mu((y+U) \cap(y+V))=0$ which is a contradiction because $(y+U)$ $\cap(y+V)$ is a neighbourhood of $y+x$. Thus $S$ satisfies the hypotheses of the above proposition so $S$ is embeddable as an open subsemigroup of a locally compact abelian group $G$. In the following we identify $S$ with its image in $G$.

Let $\mathfrak{K}_{S}(G)$ be the continuous complex-valued functions on $G$ which are zero outside of $S$ and have compact support. Observe that the invariance property of $\mu$ means that if $f \in \mathcal{K}_{S}(G)$ then for each $y$ in $S$,

$$
\int_{S} f(x-y) d \mu(x)=\int_{S} f d \mu .
$$

Choose $g \in \varkappa_{S}(G)$ with $g \geqq 0$ and $\int g d \lambda=1$ where $\lambda$ is the Haar measure on $G$. Then using the Fubini Theorem $[3$, p. 153] we have

$$
\begin{aligned}
\int_{S} f d \mu & =\int_{S} f(x-y) d \mu(x) \int_{G} g(y) d \lambda(y) \\
& =\int_{S} \int_{G} f(x-y) g(y) d \lambda(y) d \mu(x) \\
& =\int_{S} \int_{S} f(y) g(x-y) d \lambda(y) d \mu(x) \\
& =\int_{S} g(x-y) d \mu(x) \int_{S} f(y) d \lambda(y) \\
& =c \int_{S} f d \lambda
\end{aligned}
$$

where $c=\int_{S} g d \mu$. It follows now that for any Borel subset $E \subset S$, $\mu(E)=c \lambda(E)[3$, p. 129]. This completes the proof of the first statement.

If $S$ is an open subsemigroup of a locally compact abelian group, then it is clear that $S$ is a locally compact abelian semigroup. More- 
over since $S$ is open the restriction of the Haar measure on $G$ to $S$ yields a nonnegative regular measure $\mu$ such that condition $\left(^{*}\right)$ is satisfied.

\section{REFERENCES}

1. N. Bourbaki, Livre III : Topologie génêrale. Chaps. 1, 2, Actualités Sci. Indust., no. 1142, Hermann, Paris, 1965. MR 39 \#6237.

2. R. Ellis, Locally compact transformation groups, Duke Math. J. 24 (1957), 119125. MR 19, 561.

3. E. Hewitt and K. A. Ross, Abstract harmonic analysis. Vol. I: Structure of topological groups. Integration theory, group representations, Die Grundlehren der math. Wissenschaften, Band 115, Academic Press, New York; Springer-Verlag, Berlin, 1963. MR 28 \#158.

4. N. Rothman, Embedding of topological semigroups, Math. Ann. 139 (1960), 197-203. MR $22 \# 6871$.

5. J. H. Williamson, Harmonic analysis on semigroups, J. London Math. Soc. 42 (1967), 1-41. MR 34 \#8101.

McGill University, Montreal, Quebec, Canada 